\title{
NUMERICAL EXPERIMENTS CONCERNING THE EIGENVALUES OF THE LAPLACIAN ON A ZOLL SURFACE
}

\author{
GERALD R. CHACHERE
}

\section{Introduction}

A Zoll surface is a surface of revolution in $\Re^{3}$ diffeomorphic to $S$, the unit 2-sphere, with the property that all geodesics are closed with period $2 \pi . S$ is, for example, a Zoll surface. O. Zoll [7] first proved the existence of a Zoll surface not isometric to $S$.

Eigenvalues of $\Delta$, the Laplacian defined on functions whose domain is $S$, are $l(l+1)$ with multiplicities $2 l+1(l=0,1,2, \cdots)$. For Zoll surfaces other than $S$, each multiple eigenvalue $l(l+1)$ splits into a "cluster" of eigenvalues near $l(l+1)$. Specifically, a result of Alan Weinstein [5] states that there is a number $M>0$ independent of $l$ such that the eigenvalues of the $l$-th cluster are contained in the interval $[l(l+1)-M, l(l+1)+M]$.

In this work the following question is addressed: for a Zoll surface what is the structure of the $l$-th cluster for large $l$ ? Numerical experiments were made where by the eigenvalues of the first 15 or 20 clusters of selected Zoll surfaces were approximated. These computations led to two conjectures. Conjecture 1: the arithmetic mean of the eigenvalues in the $l$-th cluster approaches $l(l+1)$ as $l$ goes to $\infty$. Conjecture 2: the distribution of eigenvalues in the $l$-th cluster approaches (in a sense to be made clear) a limiting function as $l$ goes to $\infty$.

In an attempt to explain these experimental results Weinstein [6] proved a theorem. The theorem which concerns the cluster structure of $\Delta$ plus a potential function tends to corroborate the conjecture.

In $\$ 1$ a method for constructing one parameter families of Riemannian manifolds isometric to Zoll surfaces is given. In $\$ 2$ the Laplace-Beltrami operators corresponding to the Riemannian manifolds of $\S 1$ are written in geographic coordinates, and by separation of variables an ordinary differential operator $D_{\varepsilon}^{m}$ is defined. In $\S 3$ cluster is defined, and information about eigenvalues of $D_{0}^{m}$ and $\Delta$ is given. In $\S 4$ the method by which the eigenvalues 
are approximated is given. Conjectures 1 and 2 are stated exactly in $\$ 5$. In $\$ 6$ the numerical experiments are described. $\$ 7$ contains information about the accuracy of the approximations. In $\S \S 8$ and 9 evidence for conjectures 1 and 2 are given, respectively. In $\$ 10$ results for non-Zoll experiments is presented. In the appendix EIGEN 2, the subroutine that computed the elements of the matrices which approximate the $D_{\varepsilon}^{m}$ 's is described and listed.

The eigenvalues computed in the experiments do not appear in this work, but the author will send a list of these eigenvalues upon request.

We thank Alan Weinstein for suggesting these experiments.

\section{Construction of one-parameter families of Zoll metrics}

A Riemannian metric $g$ is a Zoll metric if the Riemannian manifold $(S, g)$ is isometric to a Zoll surface. We will now give a method for constructing one-parameter families of Zoll metrics $g_{\varepsilon}$ such that $g_{0}$ is the canonical metric on $S$.

Consider the map $X:[0,2 \pi] \times[0, \pi] \rightarrow \mathcal{R}^{3}$ given by

$$
(v, u) \rightarrow(\sin u \cos v, \sin u \sin v, \cos u) .
$$

$S$ is the image of $X$. Let $U \subset S$ be the image of $(0,2 \pi) \times(0, \pi)$ under $X . U$ is open and dense in $S$. Define functions $\phi$ and $\theta$ on $U$ as follows: $X(\phi(m), \theta(m))=m$ for $m \in U .(\phi, \theta)$ is the geographic coordinate system on $U ; \phi(m)$ is the longitude of $m$, and $\theta(m)$ is the latitude of $m$ measured from the "north pole".

A smooth function $\sigma:[-1,1] \rightarrow \Re$ which is zero at the end points will be called a perturbing function. For each $\varepsilon$ in some neighborhood of zero let $h_{e}$ be a positive valued function on $[0, \pi]$ given by

$$
h_{e}(\theta)=1+\varepsilon \sigma(\cos \theta) .
$$

Given a perturbing function $\sigma$ and allowing $\varepsilon$ to range over an appropriate neighborhood of zero we define a one-parameter family $g_{\varepsilon}$ of metrics on $U$ :

$$
g_{\varepsilon}=h_{\varepsilon}^{2}(\theta) d \theta^{2}+\sin ^{2} \theta d \phi^{2} .
$$

A. L. Besse [1] states that $g_{\varepsilon}$ can be extended to a metric on $S$ if the function $h_{\varepsilon}$ has the following properties: $h_{\varepsilon}$ is smooth on $[0, \pi]$, and $h_{\varepsilon}(0)=$ $h_{\varepsilon}(\pi)=1 . h_{\varepsilon}$ has those properties. Also Besse shows that if the perturbing function $\sigma$ is odd, that is, $\sigma(-x)=-\sigma(x)$, then $g_{\varepsilon}$ is a Zoll metric.

Thus $g_{\varepsilon}$ as defined by (*) is a family of metrics on $S$ for arbitrary $\sigma$, and is a family of Zoll metrics if $\sigma$ is an odd function. 


\section{Zoll perturbations of the Laplace-Beltrami operator}

For each Riemannian manifold there is a unique Laplace-Beltrami operator. We will call this operator the Laplacian, and its domain is the set of smooth function on the manifold. The Laplacian on $\Re^{2}$ with the canonical metric is

$$
-\left(\frac{\partial^{2}}{\partial x^{2}}+\frac{\partial^{2}}{\partial y^{2}}\right)
$$

We are interested in certain perturbations of $\Delta$, the Laplacian on $\left(S, g_{0}\right)$. Given $\sigma$, a perturbing function, define $\Delta_{\varepsilon}$, a perturbation of $\Delta$, to be the Laplacian on $\left(S, g_{\varepsilon}\right) \cdot g_{\varepsilon}$ is defined by $(*)$ of $\S 1$ with perturbation function $\sigma$. If $\sigma$ is odd, then we call $\Delta_{\varepsilon}$ a Zoll perturbation of $\Delta$. Thus a Zoll perturbation of $\Delta$ is generated by a Zoll metric.

If $F$ is a smooth function on $S$, then $\Delta_{\varepsilon}$ in geographic coordinates is given by

$$
\Delta_{\varepsilon}[F]=\frac{-1}{h_{\varepsilon}^{2}(\theta)} \frac{\partial^{2} F}{\partial \theta^{2}}+\left(\frac{h_{\varepsilon}^{\prime}(\theta)}{h_{\varepsilon}^{3}(\theta)}-\frac{\cot \theta}{h_{\varepsilon}^{2}(\theta)}\right) \frac{\partial F}{\partial \theta}+\frac{-1}{\sin ^{2} \theta} \frac{\partial^{2}}{\partial \phi^{2}} .
$$

This result can be seen by using the definition of $\Delta_{e}$ given by Warner [4].

For each integer $m$ we will define $D_{\varepsilon}^{m}$, a second order ordinary differential operator. The domain of $D_{\varepsilon}^{m}$ is the set of smooth functions $f$ on $[0, \pi]$ :

$$
D_{\varepsilon}^{m}[f](\theta)=\frac{-1}{h_{\varepsilon}^{2}(\theta)} f^{\prime \prime}(\theta)+\left(\frac{h_{\varepsilon}^{\prime}(\theta)}{h_{\theta}^{3}(\theta)}-\frac{\cot \theta}{h_{\varepsilon}^{2}(\theta)}\right) f^{\prime}(\theta)+\frac{m^{2}}{\sin ^{2} \theta} f(\theta) .
$$

Theorem. $\lambda$ is an eigenvalue of $\Delta_{\varepsilon}$ if and only if there is an $m$ such that $\lambda$ is an eigenvalue of $D_{\varepsilon}^{m}$.

A proof can be constructed by using two facts. One fact is that if $F$ is smooth on $S$, then $F$ is the (possibly infinite) sum of terms of the form $u_{m}(\phi) f(\theta) . f(\theta)$ is smooth on $[0, \pi]$, and

$$
u_{m}(\phi)=\left\{\begin{array}{cc}
\sin (m \phi) & \text { if } m<0 \\
1 & \text { if } m=0 \\
\cos (m \phi) & \text { if } m>0
\end{array}\right.
$$

The other fact is that

$$
\Delta_{\varepsilon}[F]=u_{m}(\phi) D_{\varepsilon}^{m}[f][\theta]
$$

if $F=u_{m}(\phi) f(\theta)$.

By means of the above theorem we will investigate the eigenvalues of $\Delta_{e}$ by examining the eigenvalues of the $D_{\varepsilon}^{m}$. 
There are two notable facts about the boundary conditions of the eigenfunctions of $D_{\varepsilon}^{m}:$ (1) if $m \neq 0$, the eigenfunctions are zero at the end points, and (2) the derivatives of the eigenfunctions of $D_{\varepsilon}^{0}$ are zero at the end points. To prove the first fact we assume that $f$ is an eigenfunction of $D_{\varepsilon}^{m}(m \neq 0)$, and obtain the followirig:

$$
\lambda f(\theta) \sin ^{2} \theta=D_{\varepsilon}^{m}[f](\theta) \sin ^{2} \theta .
$$

Expanding the right side of the above equation we obtain

$$
\left(\frac{-\sin ^{2} \theta}{h_{e}^{2}(\theta)}\right) f^{\prime \prime}(\theta)+\left(\frac{h_{\varepsilon}^{\prime}(\theta) \sin ^{2} \theta}{h_{\varepsilon}^{3}(\theta)}-\frac{\cos \theta \sin \theta}{h_{e}^{2} \theta}\right) f^{\prime}(\theta)+m^{2} f(\theta) .
$$

Therefore $f(\theta)=0$ when $\theta$ equals 0 or $\pi$.

To prove the second fact we assume that $f$ is an eigenfunction of $D_{e}^{0}$, and obtain an expression similar to the above:

$$
\lambda f(\theta) \sin \theta=D_{\varepsilon}^{0}[f](\theta) \sin \theta .
$$

We expand the right side of the above equation

$$
\left(\frac{-\sin \theta}{h_{\varepsilon}^{2}(\theta)}\right) f^{\prime \prime}(\theta)+\left(\frac{h_{\varepsilon}^{\prime}(\theta) \sin \theta}{h_{\varepsilon}^{3}(\theta)}-\frac{\cos \theta}{h_{\varepsilon}^{2}(\theta)}\right) f^{\prime}(\theta) .
$$

Therefore $f^{\prime}(\theta)=0$ when $\theta$ equals 0 or $\pi$.

It should also be noted that $D_{\varepsilon}^{m}=D_{\varepsilon}^{-m}$.

\section{Cluster of eigenvalues}

Henceforth $m$ is an integer, and $k$ and $l$ are nonnegative integers. We make the following definitions:

$$
\begin{aligned}
\mu(l) & =l(l+1), \\
\lambda_{\varepsilon}(k) & =\text { the } k \text {-th eigenvalue of } \Delta_{e}, \\
\lambda_{e}(k, m) & =\text { the } k \text {-th eigenvalue of } D_{e}^{m} .
\end{aligned}
$$

Vilenkin [3] treats the eigenvalues and eigenfunctions of $D_{0}^{m}$. Letting $x=\cos \theta$, we can write $D_{0}^{m}$ as

$$
\left(x^{2}-1\right) \frac{d^{2}}{d x^{2}}+2 x \frac{d}{d x}+\frac{m^{2}}{1-x^{2}} \text {. }
$$

The associated Legendre function $P_{l}^{m}(x)(|m| \leqslant l)$ is the eigenfunction of $D_{0}^{m}$ with value $\mu(l)$, and $\mu(l)$ is a simple eigenvalue. Thus $\lambda_{0}(k, m)=\mu(k+|m|)$.

The eigenvalues of $\Delta_{0}$ are $\mu(l)$, and have multiplicity $2 l+1$. The eigenfunctions of $\Delta_{0}$ belonging to $\mu(l)$ are $u_{m}(\phi) P_{l}^{m}(\cos \theta)$, where $|m| \leqslant l$. 
Below are listed the first few members of the spectrum of the specified operators.

$$
\begin{aligned}
& \Delta_{0}: \quad 0, \quad 2, \quad 2, \quad 2, \quad 6, \quad 6, \quad 6, \quad 6, \quad 6, \quad 12, \quad 12, \cdots \\
& D_{0}^{0}: \quad 0, \quad 2, \quad 6, \quad 12, \quad 20, \quad 30, \quad 42, \quad 56, \quad 72, \quad \ldots \\
& D_{0}^{3}: \quad 12, \quad 20,30,42,56,72,90,110, \ldots \\
& D_{0}^{-2}: \quad 6, \quad 12, \quad 20,30,42, \quad 56,72, \quad 90, \ldots .
\end{aligned}
$$

From the above we see that if $l^{2} \leqslant k<(l+1)^{2}$, then $\lambda_{0}(k)=\mu(l)$. Define the l-th order cluster of $\Delta_{\varepsilon}$ to be $\left\{\lambda_{\varepsilon}(k): l^{2} \leqslant k<(l+1)^{2}\right\} . \mu(l)$ is the only member of the $l$-th order cluster of $\Delta_{0}$.

Define the l-th cluster of $\Delta_{\varepsilon}$ to be $\left\{\lambda_{\varepsilon}(k, m): l=k+|m|\right\} . \mu(l)$ is the only member of the $l$-th cluster of $\Delta$. As $\Delta_{\varepsilon}$ is perturbed from $\varepsilon=0, \mu(l)$ splits into the eigenvalues of the $l$-th cluster.

Both the $l$-th cluster and the $l$-th order cluster are subsets of spectrum of $\Delta_{e}$, and are equal when $\varepsilon=0$. If $\varepsilon \neq 0$, the two subsets may not be equal; the clusters may "over lap", where as order clusters never "over lap".

A result of A. Weinstein [5] states the following: if $\Delta_{\varepsilon}$ is a Zoll perturbation, then there is an $M>0$ such that for all $l$ the $l$-th order cluster is contained in the interval $[\mu(l)-M, \mu(l)+M]$, where $M$ depends only on $g_{e}$. Using this result one can show that eventually the order clusters are equal to the clusters when $\Delta_{e}$ is a Zoll perturbation.

The purpose of this paper is to study the structure of the eigenvalues in the $l$-th cluster of $\Delta_{\varepsilon}$, a Zoll perturbation, as $l$ approaches $\infty$.

\section{The method of approximating eigenvalues}

In this section we give the method by which we numerically approximate the eigenvalues of $\Delta_{\varepsilon}$. To achieve our goal we actually compute approximations to the eigenvalues of $D_{\varepsilon}^{m}$.

The first step in our method is to define $E_{n}$ for $D_{\varepsilon}^{m}$, where $n$ is a positive integer. $E_{n}$ is an $(n-1) \times(n-1)$ matrix if $m \neq 0$, and $E_{n}$ is an $(n+1) \times$ $(n+1)$ matrix if $m=0$. Define $\Lambda_{\varepsilon}^{n}(k, m)$ to be the $k$-th eigenvalue of $E_{n}(k<n) . E_{n}$ will have the following important property:

$$
\lim _{n \rightarrow \infty} \Lambda_{\varepsilon}^{n}(k, m)=\lambda_{\varepsilon}(k, m) .
$$

We will approximate $\lambda_{\varepsilon}(k, m)$ by $\Lambda_{\varepsilon}^{n}(k, m)$ using large values of $n$. We will, in general, calculate the first 15 or 20 eigenvalues of $E_{n}$, for $n=1000$ or $n=2000$. 
The method of finite centered difference is used to define $E_{n}$. We divide $[0, \pi]$ into $n$ subintervals of equal length; $\theta_{i}$ 's are the end points of the subintervals. The $(n+1)$ vector $\left(f_{0}, f_{1}, f_{2}, \ldots, f_{n}\right)$ approximates $f$, an eigenfunction of $D_{\varepsilon}^{m}$, by having $f_{i} \sim f\left(\theta_{i}\right)$. $f^{\prime}\left(\theta_{i}\right)$ and $f^{\prime \prime}\left(\theta_{i}\right)$ are approximated by

$$
f^{\prime}\left(\theta_{i}\right) \sim \frac{f_{i+1}-f_{i-1}}{2 \delta}, \quad f^{\prime \prime}\left(\theta_{i}\right) \sim \frac{f_{i+1}-2 f_{i}+f_{i-1}}{\delta^{2}},
$$

where $\delta$ is the mesh size, the length of the subintervals. We now proceed formally to define $E_{n}$. Define the following expressions:

$$
\begin{aligned}
A(\theta) & =m^{2} / \sin ^{2} \theta, \\
B(\theta) & =h_{\varepsilon}^{\prime}(\theta) / h_{\varepsilon}^{3}(\theta)-\cot \theta / h_{\varepsilon}^{2}(\theta), \\
C(\theta) & =-1 / h_{\varepsilon}^{2}(\theta), \\
\theta_{i} & =i \pi / n \quad(i=0,1, \cdots, n), \\
\delta & =\pi / n=\text { the mesh size, } \\
A_{i} & =A\left(\theta_{i}\right), \quad B_{i}=B\left(\theta_{i}\right), \quad C_{i}=C\left(\theta_{i}\right) .
\end{aligned}
$$

$A(\theta), B(\theta)$, and $C(\theta)$ are the coefficients of $f(\theta), f^{\prime}(\theta)$, and $f^{\prime \prime}(\theta)$, respectively, in the definition of $D_{\varepsilon}^{m}[f](\theta)$.

Set up difference equations for $i=1, \cdots, n-1$ :

$$
A_{i} f_{i}+B_{i} \frac{f_{i+1}-f_{i-1}}{2 \delta}+C_{i} \frac{f_{i+1}-2 f_{i}+f_{i-1}}{\delta^{2}}=\lambda f_{i} .
$$

The left side of the equation approximates $D_{\varepsilon}^{m}[f]\left(\theta_{i}\right)$. The right approximates $\lambda f\left(\theta_{i}\right)$. By combining like terms the equation reduces to

$$
\alpha_{i} f_{i-1}+\beta_{i} f_{i}+\gamma_{i} f_{i+1}=\lambda f_{i} \text {, }
$$

where

$$
\alpha_{i}=\frac{C_{i}}{\delta^{2}}-\frac{B_{i}}{2 \delta}, \quad \beta_{i}=A_{i}-2 \frac{C_{i}}{\delta^{2}}, \quad \gamma_{i}=\frac{C_{i}}{\delta^{2}}+\frac{B_{i}}{(2 \delta)} .
$$

Call (**) the $i$-th difference equation.

At this point we must consider two cases for $D_{e}^{m}: m \neq 0$ and $m=0$. We proceed with case $m \neq 0$. As mentioned, $f(0)=f(\pi)=0$, when $f$ is a eigenfunction of $D_{\varepsilon}^{m}$ and $m \neq 0$. So define $f_{0}=f_{n}=0$.

Because $f_{0}=0$ the first difference equation is

$$
\beta_{1} f_{1}+\gamma_{1} f_{2}=\lambda f_{1} \text {, }
$$

and because $f_{n}=0$ the $(n-1)$-st difference equation is

$$
\alpha_{n-1} f_{n-2}+\beta_{n-1}=\lambda f_{n-1} \text {. }
$$


Using matrix notation we write the $(n-1)$ difference equations as follows:

$$
\left[\begin{array}{ccccccc}
\beta_{1} & \gamma_{1} & 0 & & & & \\
\alpha_{2} & \beta_{2} & \gamma_{2} & & & 0 & \\
0 & \alpha_{3} & \beta_{3} & & & & \\
& & & \ddots & & & \\
& & & & \beta_{n-3} & \gamma_{n-3} & 0 \\
& 0 & & & \alpha_{n-2} & \beta_{n-2} & \gamma_{n-2} \\
& & & & 0 & \alpha_{n-1} & \beta_{n-1}
\end{array}\right]\left[\begin{array}{c}
f_{1} \\
f_{2} \\
f_{3} \\
\vdots \\
f_{n-3} \\
f_{n-2} \\
f_{n-1}
\end{array}\right]=\lambda\left[\begin{array}{c}
f_{1} \\
f_{2} \\
f_{3} \\
\vdots \\
\vdots \\
f_{n-3} \\
f_{n-2} \\
f_{n-1}
\end{array}\right] .
$$

The tridiagonal matrix is the above mentioned $E_{n}$. Thus $E_{n}$ is an $(n-1) \times$ $(n-1)$ tridiagonal matrix with $\beta_{1}, \cdots, \beta_{n-1}$ on the main diagonal, $\alpha_{2}, \cdots, \alpha_{n-1}$ on the subdiagonal, $\gamma_{1}, \cdots, \gamma_{n-2}$ on the super diagonal, and zero everywhere else. By standard approximation theory $\lim _{n \rightarrow \infty} \Lambda_{\varepsilon}^{n}(k, m)=$ $\lambda_{e}(k, m)$. (See Isaacson and Keller [2].)

Next consider the case $m=0 . f^{\prime}(0)=f^{\prime}(\pi)=0$ when $f$ is an eigenfunction of $D_{\varepsilon}^{0}$. Notice that $m=0$ implies $A(\theta)=0$. We must still approximate $f_{0}$ and $f_{n}$.

Let us calculate $D_{\varepsilon}^{0}[f](\pi)$ where $f$ is an eigenfunction. Keep in mind that $B(\theta)$ has singularities at $\theta=0$ and $\theta=\pi$.

$$
\begin{aligned}
D_{\varepsilon}^{0}[f](\pi) & =\lim _{\theta \rightarrow \pi}\left(B(\theta) f^{\prime}(\theta)\right)+C(\pi) f^{\prime \prime}(\pi) \\
& =\lim _{\theta \rightarrow \pi}\left(\frac{f^{\prime}(\theta)}{\sin \theta}\right) \cos \pi+(-1) f^{\prime \prime}(\pi) .
\end{aligned}
$$

The second line is true by the definitions of $B(\theta)$ and $C(\theta)$, and because $h_{e}(\pi)=1$ and $h_{e}^{\prime}(\pi)=0$. L'Hosptal's rule gives

$$
D_{\varepsilon}^{0}[f](\pi)=-\frac{f^{\prime \prime}(\pi)}{\cos \pi}-f^{\prime \prime}(\pi)=-2 f^{\prime \prime}(\pi) .
$$

Similar calculations yield $D_{\varepsilon}^{0}[f](0)=-2 f^{\prime \prime}(0)$. We approximate $f^{\prime \prime}(\pi)$ as follows:

$$
f^{\prime \prime}(\pi) \sim \frac{f^{\prime}(\pi)-f^{\prime}(\pi-\delta / 2)}{\delta / 2} .
$$

Because $f^{\prime}(\pi)=0$ and

$$
f^{\prime}(\pi-\delta / 2) \sim \frac{f_{n}-f_{n-1}}{\delta},
$$


we make the following approximation

$$
f^{\prime}(\pi) \sim \frac{0-\frac{f_{n}-f_{n-1}}{\delta}}{\delta / 2}=-2 \frac{f_{n}-f_{n-1}}{\delta^{2}} .
$$

Combining the above we have

$$
D_{\varepsilon}^{0}[f](\pi)=-2 f^{\prime \prime}(\pi) \sim \frac{4}{\delta^{2}} f_{n}-\frac{4}{\delta^{2}} f_{n-1} .
$$

Define the $n$-th difference equation to be

$$
-\frac{4}{\delta^{2}} f_{n-1}+\frac{4}{\delta^{2}} f_{n}=\lambda f_{n}
$$

Similarly the 0 -th difference equation is defined to be

$$
\frac{4}{\delta^{2}} f_{0}-\frac{4}{\delta^{2}} f_{1}=\lambda f_{0}
$$

If we let $q=4 / \delta^{2}, \beta_{0}=q, \gamma_{0}=-q, \alpha_{n}=-q$, and $\beta_{n}=q$, then $E_{n}$ is the $(n+1) \times(n+1)$ tridiagonal matrix

$$
\left[\begin{array}{ccccccc}
\beta_{0} & \gamma_{0} & 0 & & & & \\
\alpha_{1} & \beta_{1} & \gamma_{1} & & & 0 & \\
0 & \alpha_{2} & \beta_{2} & & & & \\
& & & \ddots & & & \\
& & & & \beta_{n-2} & \gamma_{n-2} & 0 \\
& 0 & & & \alpha_{n-1} & \beta_{n-1} & \gamma_{n-1} \\
& & & & 0 & \alpha_{n} & \beta_{n}
\end{array}\right] .
$$

Again $\lim _{n \rightarrow \infty} \Lambda_{e}^{n}(k, 0)-\lambda_{e}(k, 0)$.

The second step of our method is to calculate the eigenvalues of $E_{n}$. This step is achieved by the use of EQRTIS. EQRTIS is a FORTRAN subroutine that computes the $j$ smallest eigenvalues of a tridiagonal matrix. An important feature of EQRTIS is that it computes the eigenvalues to the precision of the computer, which is about 15 decimal places.

EQRT1S is a product of Internatiolnal Mathematical and Statistical Libraries, Inc. (IMSL) of Houston, Texas, and is a part of the University of California, Berkeley computer library.

\section{The statements of Conjecture 1 and Conjecture 2}

Recall that the $l$-th cluster of $\Delta_{\varepsilon}$ is defined to be $\left\{\lambda_{\varepsilon}(k, m): k+|m|=l\right\}$, and that, as an eigenvalue of $D_{\varepsilon}^{m}, \lambda_{\varepsilon}(k, m)$ is simple. 
The $l$-th cluster of $\Delta$ contains only $\mu(l)$. The $l$-th cluster of $\Delta_{e}$ has $2 l+1$ members, counting multiplicities.

Define $\bar{\lambda}_{\varepsilon}(l)$ to be the mean of the $l$-th cluster of $\Delta_{\varepsilon}$ :

$$
\bar{\lambda}_{\varepsilon}(l)=\frac{\sum_{m=-l}^{l} \lambda(l-|m|, m)}{2 l+1} .
$$

Conjecture 1. If $\Delta_{\varepsilon}$ is a Zoll perturbation of $\Delta$, then $\left|\mu(l)-\bar{\lambda}_{\varepsilon}(l)\right|$ approaches zero as l goes to infinity.

For the $l$-th cluster of $\Delta_{\varepsilon}$ we define a real valued function $G_{l}$ whose domain is the interval $[-1,1]$. We make the following definitions:

$$
\begin{aligned}
x_{m} & =m / l \quad(|m| \leqslant l), \\
y_{m} & =\lambda_{e}(l-|m|, m)-\mu(l), \\
G_{l}\left(x_{m}\right) & =y_{m} .
\end{aligned}
$$

If $x_{m} \leqslant x \leqslant x_{m+1}$, then

$$
G_{l}(x)=y_{m}+\frac{y_{m+1}-y_{m}}{x_{m+1}-x_{m}}\left(x-x_{m}\right) .
$$

Thus the value of. $G_{l}(x)$ when $x_{m} \leqslant x \leqslant x_{m+1}$ is found by linear interpolatiion. This definition is not valid for $G_{0}(x)$; for completeness let $G_{0}(x)=0$ for all $x$ in $[-1,1]$. (For every perturbation of $\Delta$ the 0 -th cluster is $\{\mu(0)\}$.) Because $\lambda_{\varepsilon}(k, m)=\lambda_{e}(k,-m), G_{l}$ is always an even function.

Consider $G_{3}$ for a certain perturbation of $\Delta$. We use Table 5. (Keep in mind that $\mu(3)=13$.)

\begin{tabular}{r|c|r|r}
$m$ & $\lambda_{\varepsilon}(3-|m|, m)$ & $x_{m}$ & $y_{m}$ \\
\hline 0 & 12.55 & 0 & 0.55 \\
\pm 1 & 11.77 & $\pm 1 / 3$ & -0.23 \\
\pm 2 & 11.65 & $\pm 2 / 3$ & -0.35 \\
\pm 3 & 12.22 & +1 & 0.22
\end{tabular}

TABLE 5

The graph of $G_{3}$ follows.

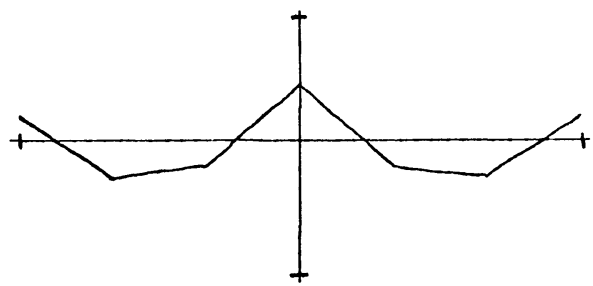


Conjecture 2. If $\Delta_{\varepsilon}$ is a Zoll perturbation of $\Delta$, then there is a function, call it $G_{\infty}$, on $[-1,1]$ such that $\left\|G_{l}-G_{\infty}\right\|$ approaches zero as $l$ goes to infinity, where $\|$ || is the sup-norm.

The result of Weinstein mentioned in $\$ 3$ implies that $G_{\infty}$ is bounded.

Clearly, both conjectures are true for $\Delta_{\varepsilon}$ when $\varepsilon=0$.

\section{Nature of the experiments}

An experiment is a set of computer calculations which approximate eigenvalues of some perturbation of $\Delta$.

In the FORTRAN program used to do the calculations the input parameters are FACTOR, IFIRST, $M, N$, and $\operatorname{SIG}(X)$. FACTOR is a number in the interval $[0,1)$. IFIRST is a positive integer. $M$ is a nonnegative integer corresponding to $|m|$, where $m$ is the parameter for the operator $D_{\varepsilon}^{m} . N$ is a positive integer corresponding to $n$, where $\pi / n$ is the mesh size. $\operatorname{SIG}(X)$ corresponds to $\sigma(x)$, the perturbing function.

$g_{\varepsilon}$, as defined by (*), is a Riemannian metric for $S$ when $\varepsilon$ is a member of a certain maximal open interval about zero. Let $\varepsilon_{0}$ be an endpoint of the interval nearest zero. This would imply that $g_{\varepsilon_{0}}$ is not a Riemann metric, and that $g_{\varepsilon}$ is when $|\varepsilon|<\left|\varepsilon_{0}\right|$. For a Zoll metric the endpoints are equi-distant from zero.'

There are two types of experiments, short and full. A short experiment uses all input parameters. The outputs are $\Lambda_{\varepsilon}^{N}(k, M)$ where $0<k<$ IFIRST, where $\varepsilon=\varepsilon_{0}(\mathrm{FACTOR})$, and where $\operatorname{SIG}(X)$ is the perturbing function for $\Delta_{\varepsilon}$. A short experiment therefore approximates the first IFIRST eigenvalues of $D_{\varepsilon}^{m}$.

In a full experiment FACTOR, IFIRST, $\mathrm{N}$, and $\operatorname{SIG}(X)$ are inputs, but $M$ is not. The outputs are $\Lambda_{e}^{n}(k, m)$, where $M \geqslant 0$ and $M+k<$ IFIRST. FACTOR and $\operatorname{SIG}(X)$ have the same role as in a short experiment. A full experiment approximates the first IFIRST clusters of $\Delta_{\boldsymbol{C}}$

Table 6 lists experiments, their types, and their inputs. Each experiment is named by a number. In an experiment with FACTOR $=0$ the eigenvalues are for $\Delta$ are approximated, and therefore $\operatorname{SIG}(X)$ is not given.

$N$ is not listed for experiment 11 . The reason being that different values of $N$ were used. The $\Lambda_{\varepsilon}^{N}(k, M)$ were calculated for $k+M<$ IFIRST $=40$. The values of $N$ are as follows: $N=2000$ for $M=0, \cdots, 30 ; N=1000$ for $M=31, \cdots, 34 ; N=500$ for $M=35, \cdots, 39$.

Zoll experiments are experiments in which $\operatorname{SIG}(X)$ is an odd function. Experiments in which FACTOR $=0$ are automatically Zoll experiments. 


\begin{tabular}{c|c|c|c|c|c|l}
$\begin{array}{c}\text { Experiment } \\
\text { Number }\end{array}$ & $\begin{array}{c}\text { Type of } \\
\text { Experiment }\end{array}$ & $N$ & $M$ & FACTOR & IFIRST & SIG $(X)$ \\
\hline 1 & full & 2000 & & 0.0 & 20 & \\
2 & full & 2000 & & 0.1 & 20 & $X^{3}-X$ \\
3 & full & 2000 & & 0.2 & 20 & $X^{3}-X$ \\
4 & full & 2000 & & 0.4 & 20 & $X^{3}-X$ \\
5 & full & 2000 & & 0.8 & 20 & $X^{3}-X$ \\
6 & full & 1000 & & 0.0 & 15 & \\
7 & full & 1000 & & 0.9999 & 15 & $X^{3}-X$ \\
8 & full & 1000 & & 0.9 & 9 & $X^{2}-1$ \\
9 & full & 2000 & & 0.4 & 20 & $\left(1-X^{2}\right)$ \\
10 & & & & & & $\cdot\left(X^{3}-.4 X\right)$ \\
11 & full & 2000 & & 0.4 & 20 & $1-X^{2}$ \\
12 & full & & & 0.4 & 40 & $X^{3}-X$ \\
13 & short & 2000 & 0 & 0.0 & 40 & \\
& full & 1000 & & 0.8 & 9 & $X^{3}-X$
\end{tabular}

TABLE 6. LIST OF EXPERIMENT AND INPUTS

\section{Considerations on the accuracy of approximations}

Two considerations contribute to our knowledge of the accuracy of our approximations to $\lambda_{e}(k, m)$.

Basically, we are interested in the truncation error, $\left|\lambda_{e}(k, m)-\Lambda_{e}^{n}(k,|m|)\right|$. When can the truncation errors be known exactly? Only when the $\lambda_{\varepsilon}(k, m)$ are known, and that is only when $\varepsilon=0$. Recall $\lambda_{0}(k, m)=\mu(k+|m|)$.

Consideration 1. In all experiments with FACTOR $=0$ the following is true:

$$
\left|\mu(l)-\Lambda_{0}^{n}(l, 0)\right|=\max \left\{\left|\mu(l)-\Lambda_{0}^{n}(k,|m|)\right|: k+|m|=l\right\} .
$$

The consideration says that among the computed approximations to the members of the $l$-th cluster of $\Delta, \Lambda_{0}^{n}(l, 0)$ has the greatest deviation from $\mu(l)$.

Consideration 2. It will be assumed that $\left|\lambda_{\varepsilon}(k, m)-\Lambda_{\varepsilon}^{n}(k,|m|)\right|$ is near $\left|\lambda_{0}(k, m)-\Lambda_{0}^{n}(k,|m|)\right|$.

Table 7 lists the errors $\mu(l)-\Lambda_{0}^{n}(l, 0)$ generated by experiments. In experiment $1, n=2000$ and $l=0, \cdots, 19$, and in experiment $6, n=1000$ and $l=0, \cdots, 14$. The table should give us an upper estimate of the truncation errors. 


\begin{tabular}{r|r|c|c} 
& & Experiment 6 & Experiment 1 \\
$l$ & $\mu(l)$ & $\mu(l)-\Lambda_{0}^{1000}(l, 0)$ & $\mu(l)-\Lambda_{0}^{2000}(l, 0)$ \\
0 & 0 & .00000 & .00000 \\
1 & 2 & .00000 & .00000 \\
2 & 6 & .00003 & .00001 \\
3 & 12 & .00011 & .00003 \\
4 & 20 & .00032 & .00008 \\
5 & 30 & .00072 & .00018 \\
6 & 42 & .00142 & .00036 \\
7 & 56 & .00254 & .00063 \\
8 & 72 & .00421 & .00105 \\
9 & 90 & .00660 & .00165 \\
10 & 110 & .00987 & .00247 \\
11 & 132 & .01423 & .00356 \\
12 & 156 & .01990 & .00497 \\
13 & 186 & .02710 & .00678 \\
14 & 210 & .03611 & 00903 \\
15 & 240 & & .00118 \\
16 & 272 & & .01516 \\
17 & 306 & & .01919 \\
18 & 342 & & .02398 \\
19 & 380 & & .02963
\end{tabular}

TABLE 7. $\mu(l)-\Lambda_{0}^{n}(l, 0)$ for Experiments 1 and 6.

\section{Evidence for Conjecture 1}

Evidence for the conjectures was generated by the Zoll experiments with FACTOR $\neq 0$. Define the computed mean,

$$
\bar{\Lambda}_{\varepsilon}^{n}(l)=\frac{\Lambda_{\varepsilon}^{n}(l)+2 \Sigma_{m-1}^{l} \Lambda_{\varepsilon}^{n}(l-m, m)}{2 l+1} .
$$

To support Conjecture 1 we will examine the computed mean deviation (CMD), $\mu(l)-\bar{\Lambda}_{e}^{n}(l)$. (Note the absence of absolute value signs.)

Table 8 lists the CMD's of the relevant experiments. In columns one and two are the cluster numbers, $l$, and the $\mu(l)$ respectively. Above the remaining columns are experiment numbers, and below the experiment numbers are the CMD's for that experiment. So, for example, to find the CMD for the 7-th cluster of experiment 5 , look in the column under 5 and on line $l=7$. 
For experiment 11 only the CMD's for $l=20, \cdots, 39$ are listed. The reason is that experiments 11 and 4 are identical for $l=0, \cdots, 19$. Therefore it would be repetitious to have CMD's listed again in the table.

Below are some observations about Table 8. The remarks after the observations are interpreted to be evidence for Conjecture 1 .

Observation 1. In each experiment, $\mu(l)-\bar{\Lambda}(l)$ is eventually very small compared to $\mu(l)$.

Remark. If Conjecture 1 is true, then $\mu(l)-\bar{\Lambda}_{e}^{n}(l)$ approaches $\bar{\lambda}_{e}(l)-$ $\bar{\Lambda}_{e}^{n}(l)$. But $\bar{\lambda}_{\varepsilon}(l)-\bar{\Lambda}_{\varepsilon}^{n}(l)$ is small.

Observation 2. A CMD of experiment 5 is closer to zero than the corresponding CMD of experiment 13. For $l=0, \cdots, 8$ these two experiments approximate the same eigenvalues, but for experiment $5, n=2000$ and for experiment $13, n=1000$.

\begin{tabular}{|c|c|c|c|c|c|c|c|c|c|c|c|}
\hline e) & $\mu$ & 2 & 3 & 4 & 9 & 5 & 13 & 7 & $\ell$ & $\mu(l)$ & 11 \\
\hline 0 & 0 & .00000 & .00000 & .00000 & .00000 & .00000 & .00000 & .00000 & 20 & 420 & .01699 \\
\hline 1 & 2 & .00085 & .00333 & .01223 & .00766 & .03558 & .03558 & .04450 & 21 & 462 & .02056 \\
\hline 2 & 6 & .00002 & .00020 & .00315 & .01 .791 & .03928 & .03931 & .07039 & 22 & 506 & .02466 \\
\hline 3 & 12 & .00001 & .00005 & .00084 & .00460 & .02097 & .02106 & .06109 & 23 & 552 & .02935 \\
\hline 4 & 20 & .00003 & .00003 & .00039 & .00190 & .02143 & .02165 & .05008 & 24 & 600 & .03466 \\
\hline 5 & 30 & .00007 & .00008 & .00020 & .00140 & .02136 & .02184 & .07565 & 25 & 650 & .04068 \\
\hline 6 & 42 & .00013 & .00014 & .00022 & .00071 & .01221 & .01310 & .07733 & 26 & 702 & .04743 \\
\hline 7 & 56 & .00029 & .00026 & .00032 & .00073 & .01611 & .01765 & .05934 & 27 & 756 & .05501 \\
\hline 8 & 72 & .00040 & .00042 & .00052 & .00060 & .01155 & .01407 & .08790 & 28 & 812 & .06364 \\
\hline 9 & 90 & .00063 & .00066 & .00078 & .00088 & .00985 & & .03069 & 29 & 870 & .07284 \\
\hline 10 & 110 & .00095 & .00099 & .00118 & .00119 & .01132 & & .07774 & 30 & 930 & .08323 \\
\hline 11 & 132 & .00136 & .00142 & .00169 & .00171 & .00878 & & .09977 & 31 & 992 & .09471 \\
\hline 12 & 156 & .00190 & .00199 & .00235 & .00237 & .00964 & & .11201 & 32 & 1056 & .10740 \\
\hline 13 & 182 & .00258 & .00270 & .00320 & .00320 & .01073 & & .10622 & 33 & 1122 & .12148 \\
\hline 14 & 210 & .00360 & .00361 & .00426 & .00425 & .01039 & & .11919 & 34 & 1190 & .13699 \\
\hline 15 & 240 & .00450 & .00471 & .00556 & .00554 & .01295 & & & 35 & 1260 & .15430 \\
\hline 16 & 272 & .00570 & .00606 & .00714 & .00709 & .01455 & & & 36 & 1332 & .17375 \\
\hline 17 & 306 & .00732 & .00766 & .00818 & .00896 & .01683 & & & 37 & 1406 & .19586 \\
\hline 18 & 342 & .00914 & .00957 & .01127 & .01118 & .02064 & & & 38 & 1482 & .22141 \\
\hline 19 & 380 & .01129 & .01181 & .01392 & .01379 & .02408 & & & 39 & 1560 & .25034 \\
\hline
\end{tabular}

TABLE 8. Computed Mean Deviations for Zoll Experiments. 
Remark. The observation is consistent with Conjecture 1 for the following reason: $\bar{\Lambda}_{\varepsilon}^{2000}(l)$ is a better approximation to $\bar{\lambda}_{\varepsilon}(l)$ that $\bar{\Lambda}_{\varepsilon}^{1000}(l)$. So, if $\left|\mu(l)-\bar{\lambda}_{\varepsilon}(l)\right|$ is near zero, then $\bar{\Lambda}_{\varepsilon}^{2000}(l)$ should be nearer to $\mu(l)$ than $\bar{\Lambda}_{\varepsilon}^{1000}(l)$.

Observation 3. In each experiment, $\mu(l)-\bar{\Lambda}_{\mathbf{e}}^{n}(l)$ is eventually less than $\mu(l)-\Lambda_{0}^{n}(l, 0)$. (See Table 7.)

Remark. $\mu(l)-\bar{\Lambda}_{\varepsilon}^{n}(l)$ approaches $\bar{\lambda}_{e}(l)-\bar{\Lambda}_{e}^{n}(l)$ by Conjecture 1. So $\mu(l)$ - $\bar{\Lambda}_{e}^{n}(l)$ approaches $\bar{\lambda}_{\mathbf{k}} 0(l)-\bar{\Lambda}_{0}^{n}(l)$ by Consideration 2. $\bar{\lambda}_{0}(l)=\mu(l)$. Therefore by Consideration 1 , eventually $\left|\mu(l)-\bar{\Lambda}_{e}^{n}(l)\right|<\left|\mu(l)-\Lambda_{0}^{n}(l, 0)\right|$.

\section{Evidence for Conjecture 2}

The evidence for Conjecture 2 is contained in the graphs which approximate some of the $G_{l}$ 's.

The graphs of this section were generated by output from Experiments 2, 3, $4,5,7,9$. Only the graphs from one experiment will be on any one page. At the top of each page will be the number of the experiment; to the left of each graph will be the cluster number. The $y$-axis is scaled so that the "top" and the "bottom" of the $y$-axis represents a certain distance from the origin. That distance is given by Table 9 .

\begin{tabular}{|l|c|c|c|c|c|c|}
\hline Experiment & 2 & 3 & 4 & 5 & 7 & 9 \\
\hline Distance & .029 & .048 & .401 & 1.052 & 2.499 & .353 \\
\hline
\end{tabular}

TABLE 9

Comments about experiments 2 and 3 should be made. The graphs of experiments 2 and 3 seem to "dip" in the center as $l$ becomes larger. The phenomenon is caused by two factors: (1) as $l$ increases the approximation to $G_{l}$ has greater error toward the center of the graph; (2) the error is exaggerated by the fact that the distance from the "bottom" of the $y$-axis to the origin is of the same order as the center errors. In experiment 2 the distance is .929 and $\mu(19)-\Lambda_{0}^{2000}(19,0)=.02963$ (see consideration 1 and 2 of Section 7). 
Experiment 2

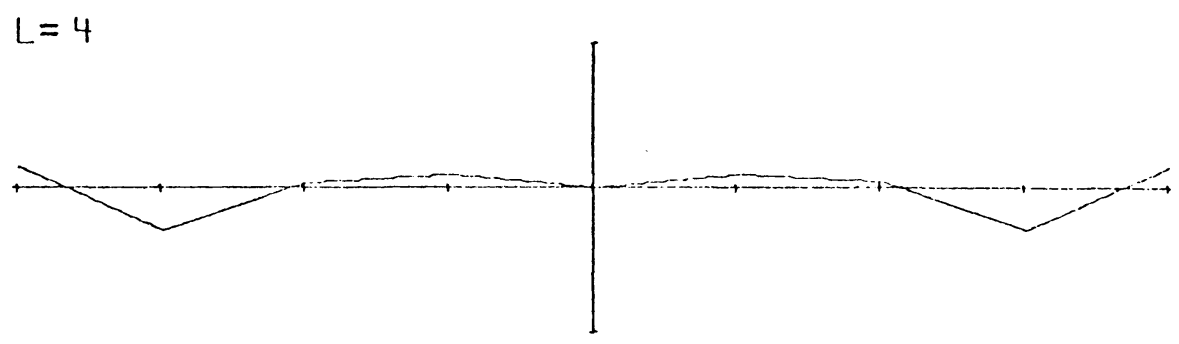

$L=9$

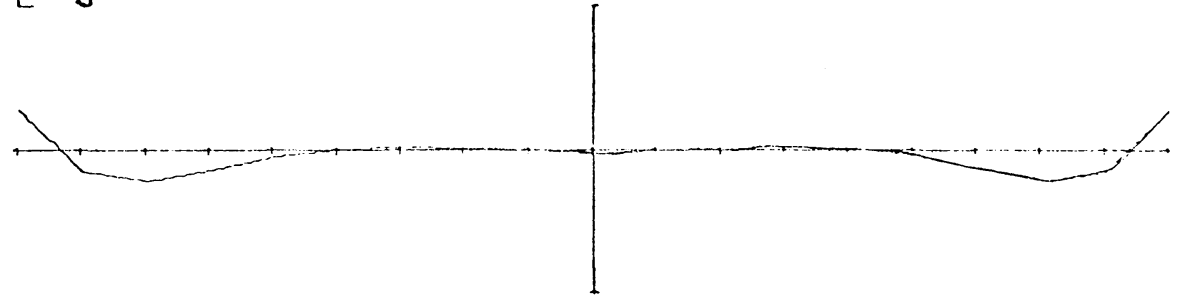

$L=14$

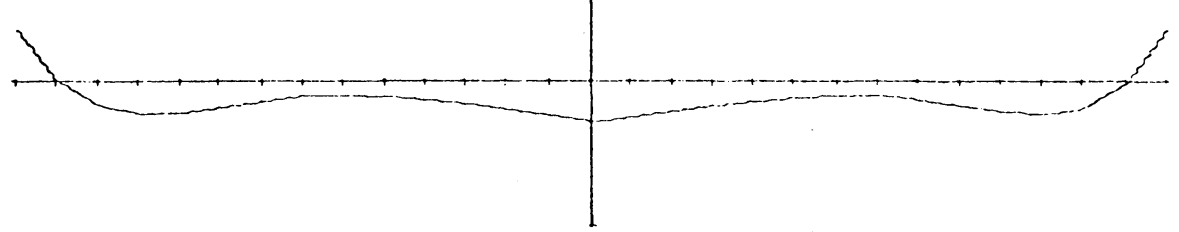

$L=19$

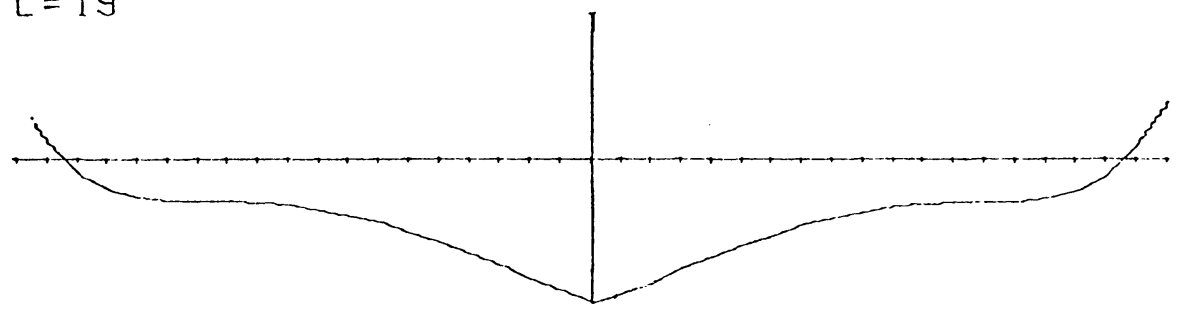


150

GERALD R. CHACHERE

Experiment 3

$$
L=3
$$

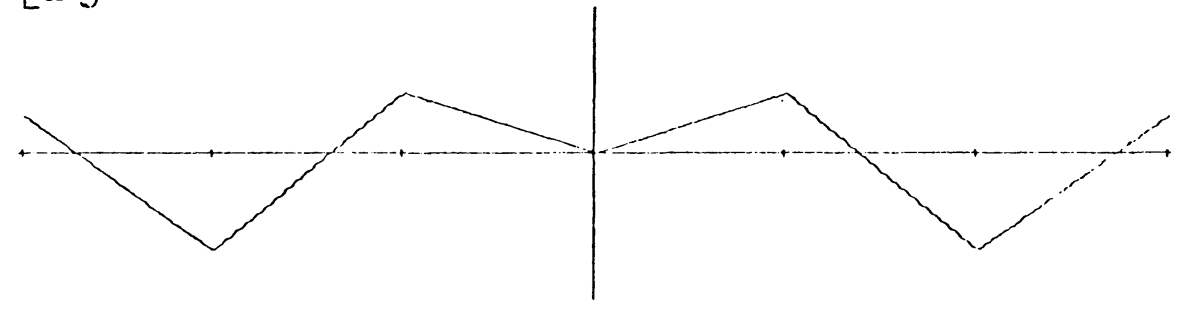

$L=7$

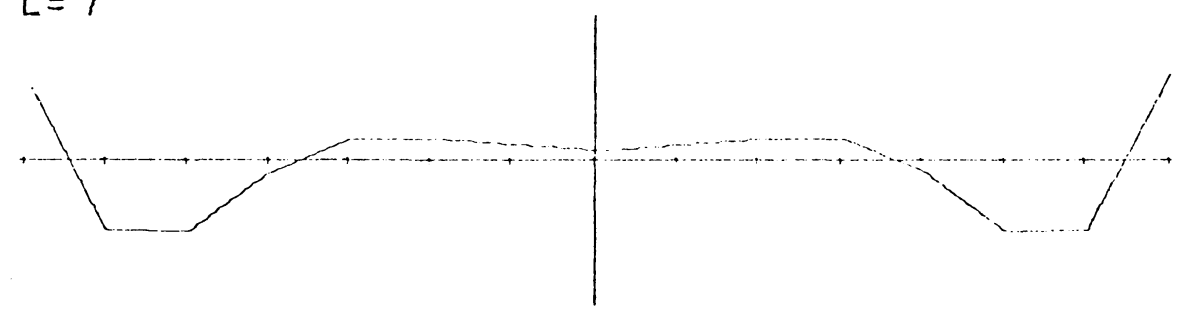

$L=11$
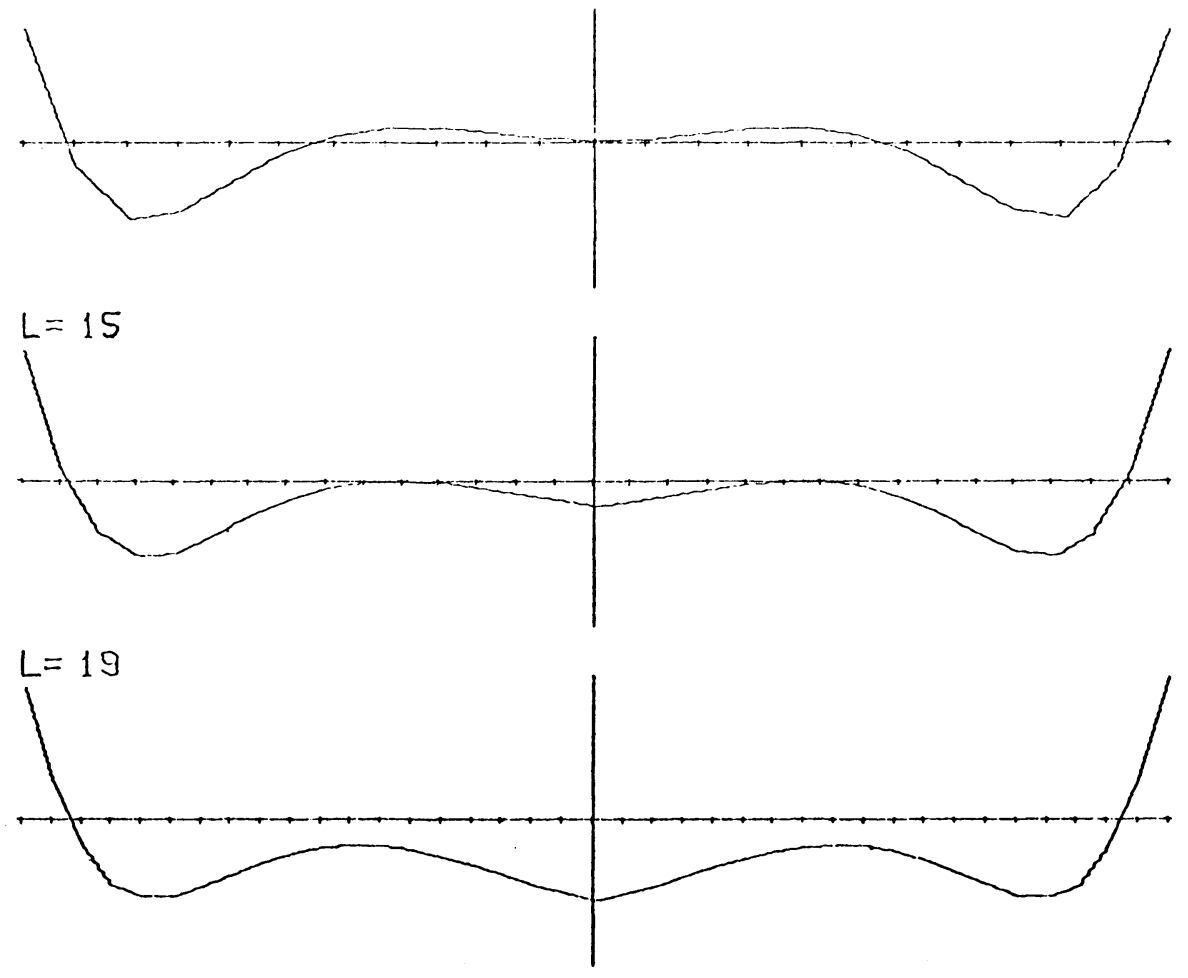
EIGENVALUES OF THE LAPLACIAN

151

Experiment 4

$L=3$

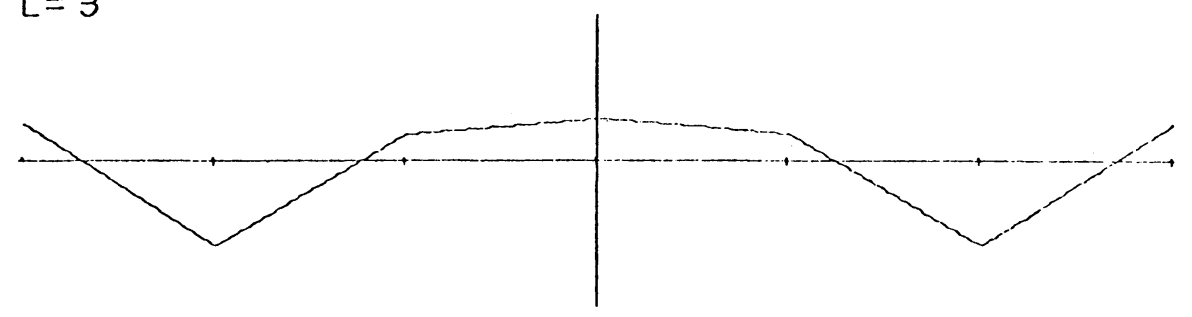

$L=7$

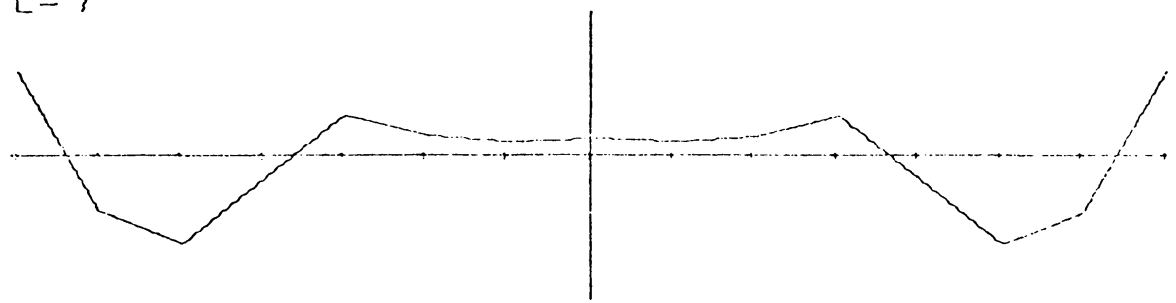

$L=11$
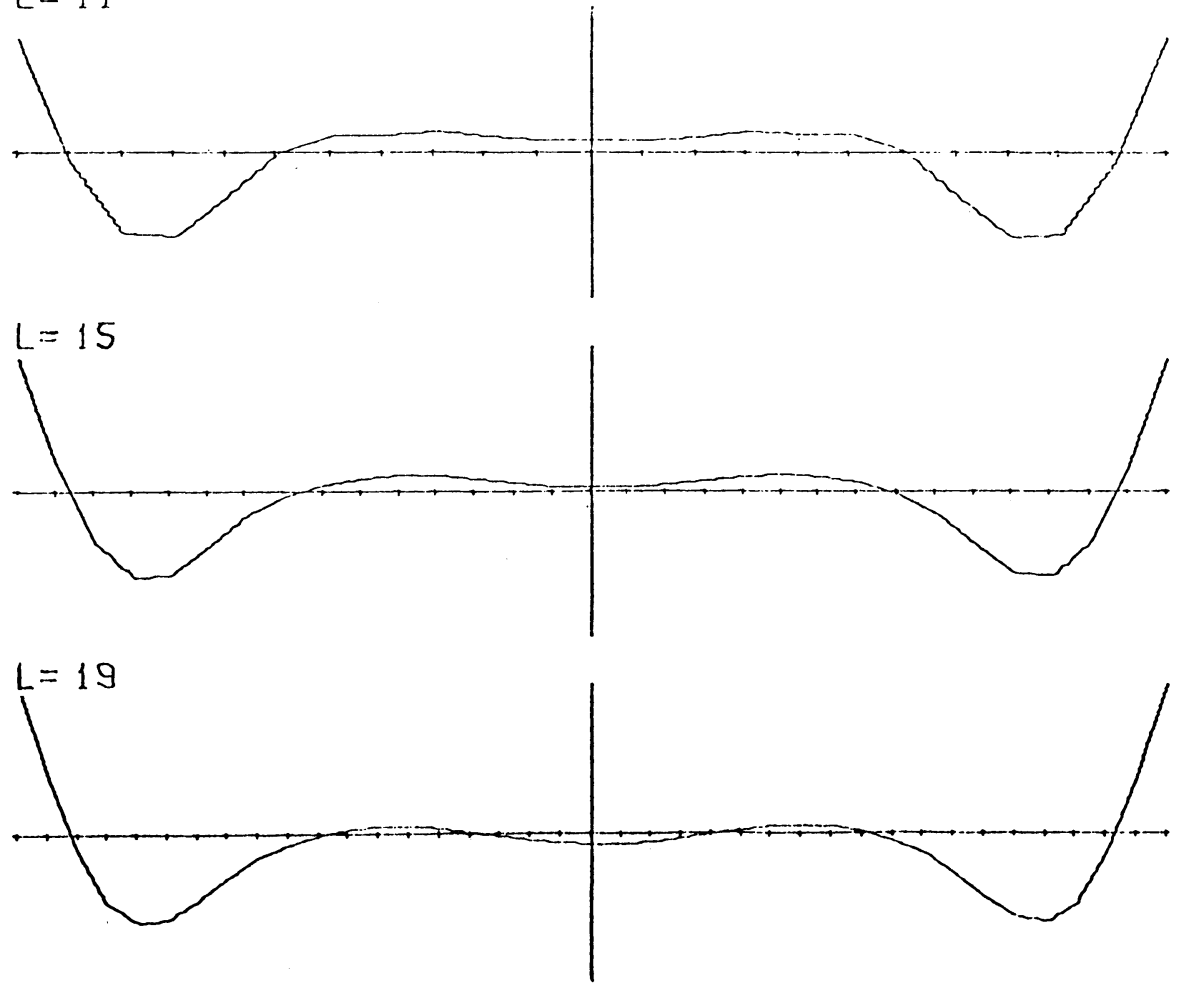
152

GERALD R. CHACHERE

Experiment 5

$L=3$

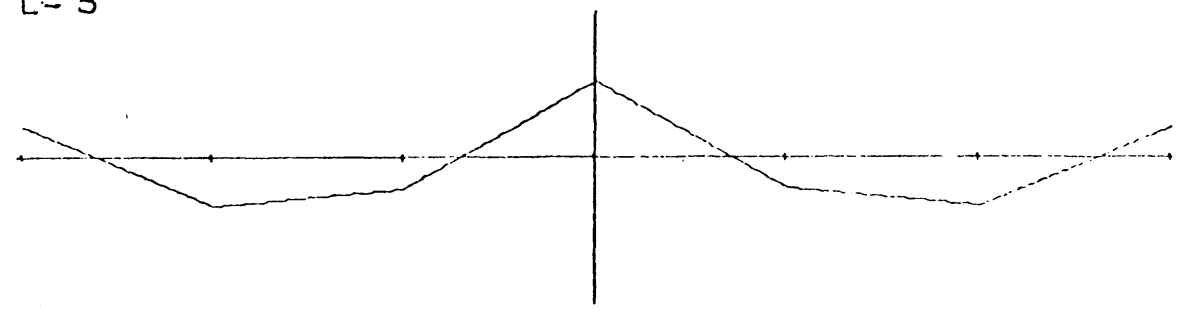

$L=7$
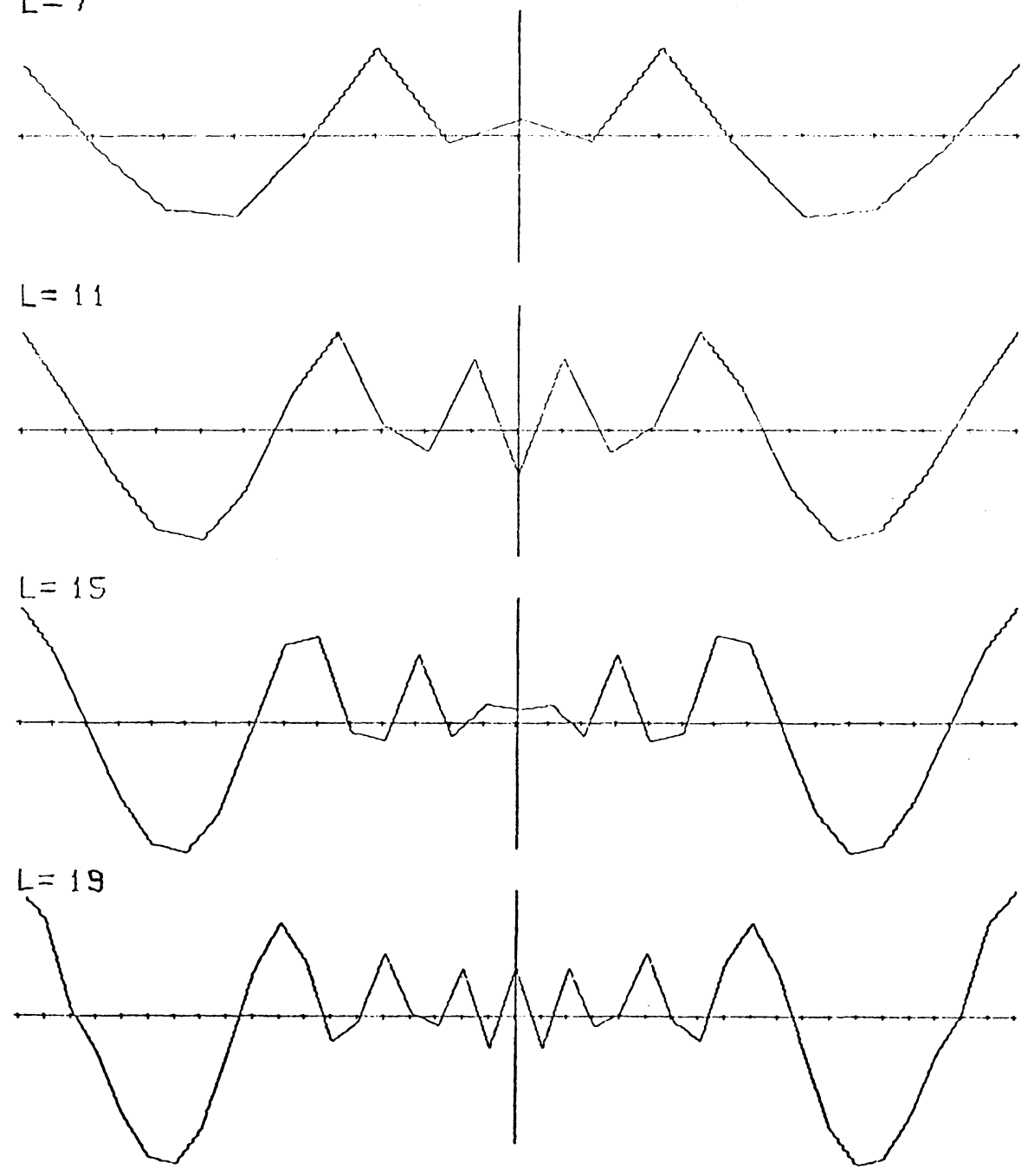


\section{Experiment 7}

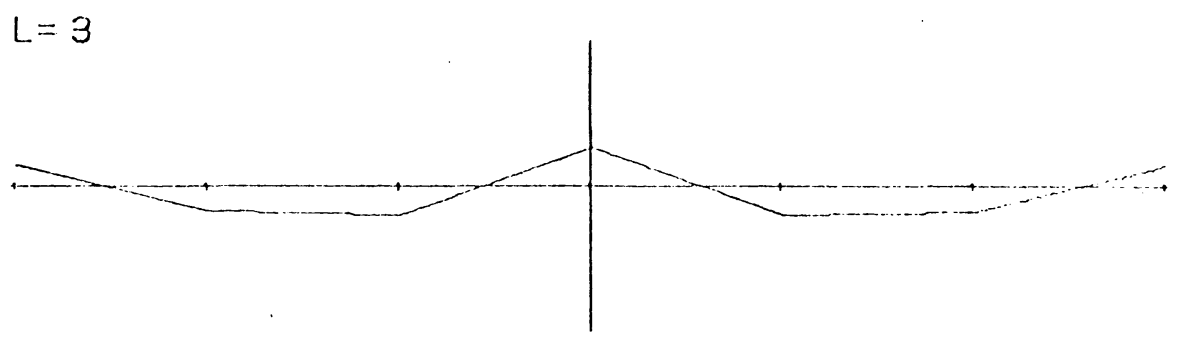

$$
L=7
$$
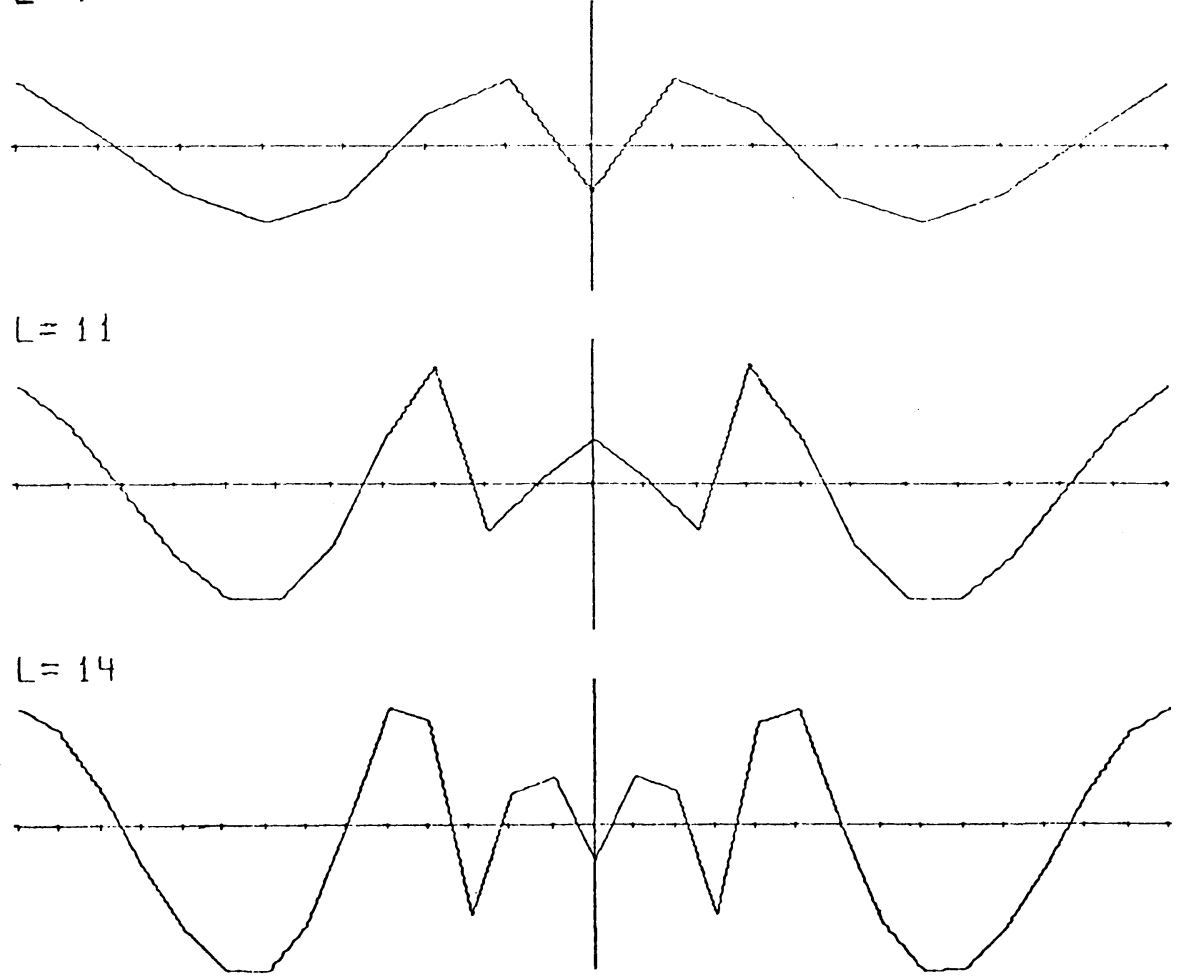
154

GERALD R. CHACHERE

Experiment 9

$L=3$

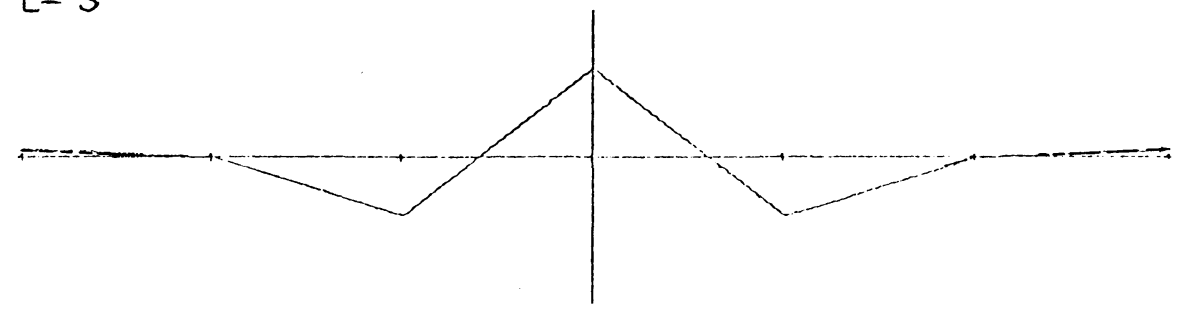

$L=7$

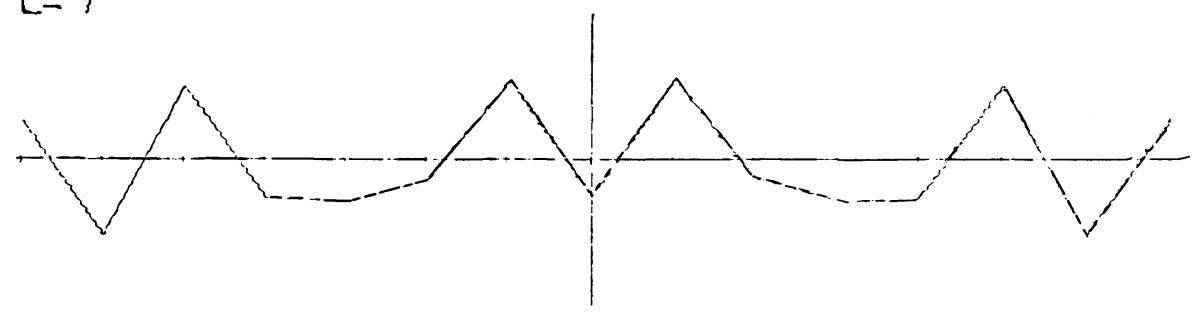

$L=11$

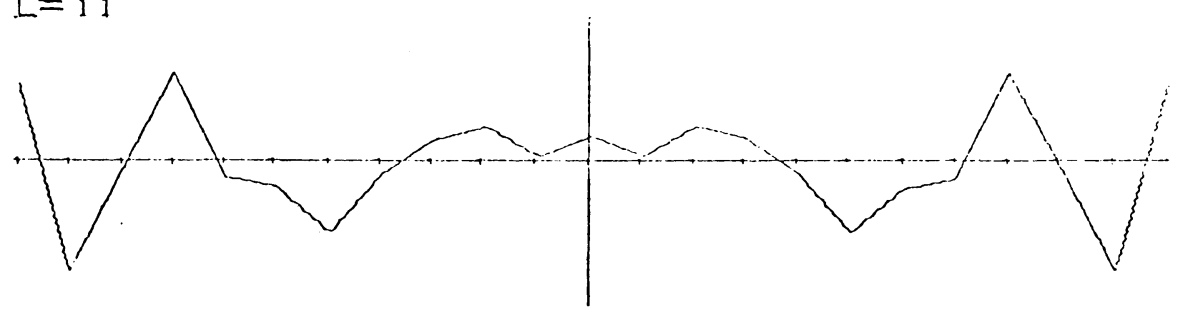

$L=15$
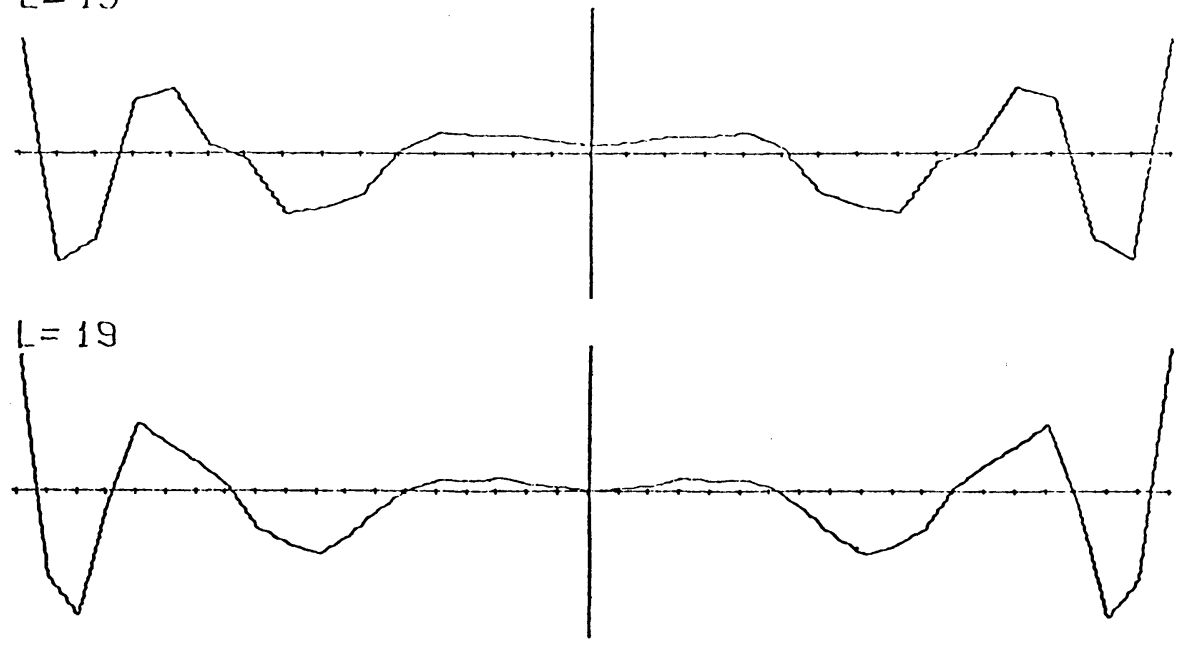


\section{Results for non-Zoll experiments}

Two non-Zoll experiments were made, 8 and 10. In both experiments the perturbing functions are even, that is, $\sigma(x)=\sigma(-x)$ for $-1 \leqslant x \leqslant 1$. Recall that $\sigma(x)$ must be odd for the perturbation of $\Delta$ to the Zoll. $x^{2}-1$ is the perturbing function for 10 , and $1-x^{2}$ for 10 .

If $\sigma(x)$ is odd, then for equal values of $\varepsilon$ the metrics induced by $\sigma(x)$ and $-\sigma(x)$ are isometric. Thus the spectrum of the corresponding Laplacians are the same. If $\sigma(x)$ is not odd, then the metric for $\sigma(x)$ and $-\sigma(x)$ are not necessarily isometric. This fact is mentioned to indicate that the Laplacians for the two non-Zoll experiments are essentially different.

The initial cluster for both 8 and 10 has only one member, .0000 . This is the same situation for Zoll experiments.

In experiment 8 for each $l>0$ the $l$-th cluster displays the following pattern:

$$
\Lambda_{e}^{n}(l, 0)<\Lambda_{e}^{n}(l-1,1)<\cdots<\Lambda_{e}^{n}(0, l)<\mu(l) .
$$

This experiment also shows that $\Lambda_{\varepsilon}^{n}(l, 0)-\mu(l, 0)-\mu(l)$ gets larger as $l$ gets larger. $\Lambda_{e}^{n}(l, 0)-\mu(l)$ gets large so fast that the clusters eventually overlap each other, that is, there is an $l_{0}$ such that for $l>l_{0}, \Lambda_{e}^{n}(l+1,0)<\Lambda_{\varepsilon}^{n}(0, l)$. In fact, the minimum member of the 8-th cluster, $\Lambda_{e}^{n}(8,0)=34.11927$, is less than the maximum member of the 6-cluster, $\Lambda_{\varepsilon}^{n}(0,6)=39.21949$.

In experiment 10 for each $l>0$ the $l$-th cluster displays the following pattern:

$$
\Lambda_{\varepsilon}^{n}(l, 0)>\Lambda_{\varepsilon}^{n}(l-1,1)>\cdots>\Lambda_{e}^{n}(0, l)>\mu(l) .
$$

This pattern is the reverse of the 8 pattern. Again the clusters eventually overlap. The maximum member of the 16-th cluster, $\Lambda_{g}^{n} \mathrm{y}(16,0)=425.12273$, is greater than the minimum of the 19-th cluster, $\Lambda_{e}^{n}(0,19)=391.90565$.

If the patterns of these two non-Zoll experiments are persistent, then the following statements would be true:

(1) the diameter of the $l$-th cluster union the set containing $\mu(l)$ is not bounded:

(2) Conjecture 1 does not hold.

\section{APPENDIX; LISTING OF SUBROUTINE EIGEN2}

What follows is a listing of SUBROUTINE EIGEN2. This subroutine is the part of the computer program which actually does computation described in §4. The language of the subroutine is FORTRAN. 
Basically this subroutine first computes the nonzero elements of the tridiagonal matrix which is to be diagonalize. $\operatorname{DIAG}(K)=\beta_{K}$ are the diagonal elements $(K=1, \cdots, N-1$ for $m \neq 0$, and $K=1, \cdots, N+1$ for $M=$ $0) \operatorname{OFF}(K)$ is the product of the corresponding elements of the super- and sub-diagonals $(K=2, \cdots, N-1$ for $M \neq 0$, and $K=2, \cdots, n+1$ for $M=0) . \operatorname{OFF}(K)=\alpha_{K} \gamma_{K-1}$.

Next, SUBROUTINE EQRET1S is called. This subroutine calculates the first IFIRST eigenvalues of the tridiagonal matrix, and stores the values in $\operatorname{DIAG}(K), K=1, \cdots$, IFIRST.

In this particular version $\operatorname{SIG}(X)=1-X^{2}$. To make experiments with different perturbing functions $\operatorname{SIG}(X)$ and its derivative, $\operatorname{DSIG}(X)$ must be defined by the appropriate functions.

\section{SUBROUTINE EIGEN2}

C

C INPUT N = NUMBER OF EQUAL SUBDIVISIONS

C $\quad M=$ DIFF. EQ. PARAMETER $=0,1,2,3, \cdots$

C EPSILON = DIFF. EQ. PARAMETER NEAR ZERO

C IFIRST $=$ NUMBER OF EIGENVALUES TO BE COMPUTED

C OUTPUT DIAG(K) = K-TH EIGENVALUE $(\mathrm{K}=1$, IFIRST)

COMMON N, M, EPSILON, DIAG(5000), IFIRST

DIMENSION OFF (5000)

$\operatorname{SIG}(X)=1-X * X$

$\operatorname{DSIGN}(X)=-2 * X$

C

C DEFINE DIAG, OFF

C

C

PI $=3,141592653589793$

GAMMA $=0$

$\mathrm{N} 1=\mathrm{N}-1$

DELTA $=$ PI $/ N$

DO $10 \mathrm{~K}=1, \mathrm{~N} 1$

THETA $=\mathrm{K} *$ DELTA

STHETA $=$ SIN(THETA)

CTHETA $=$ COS(THETA)

$\mathrm{H}=1$ - EPSILON*SIG(CTHETA)

DH $=$ EPSILON $*$ DSIG $($ CTHETA $) *$ STHETA 


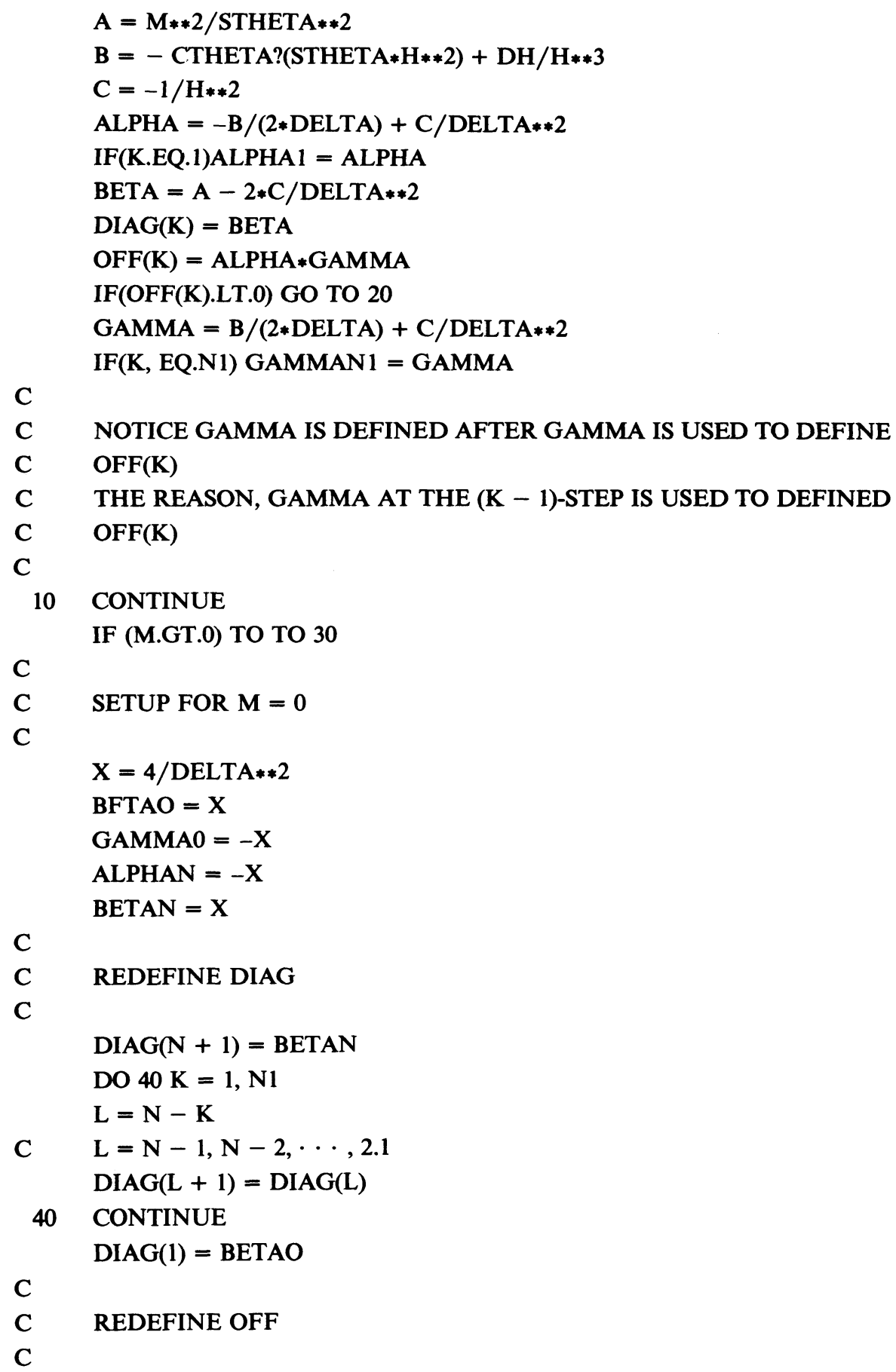




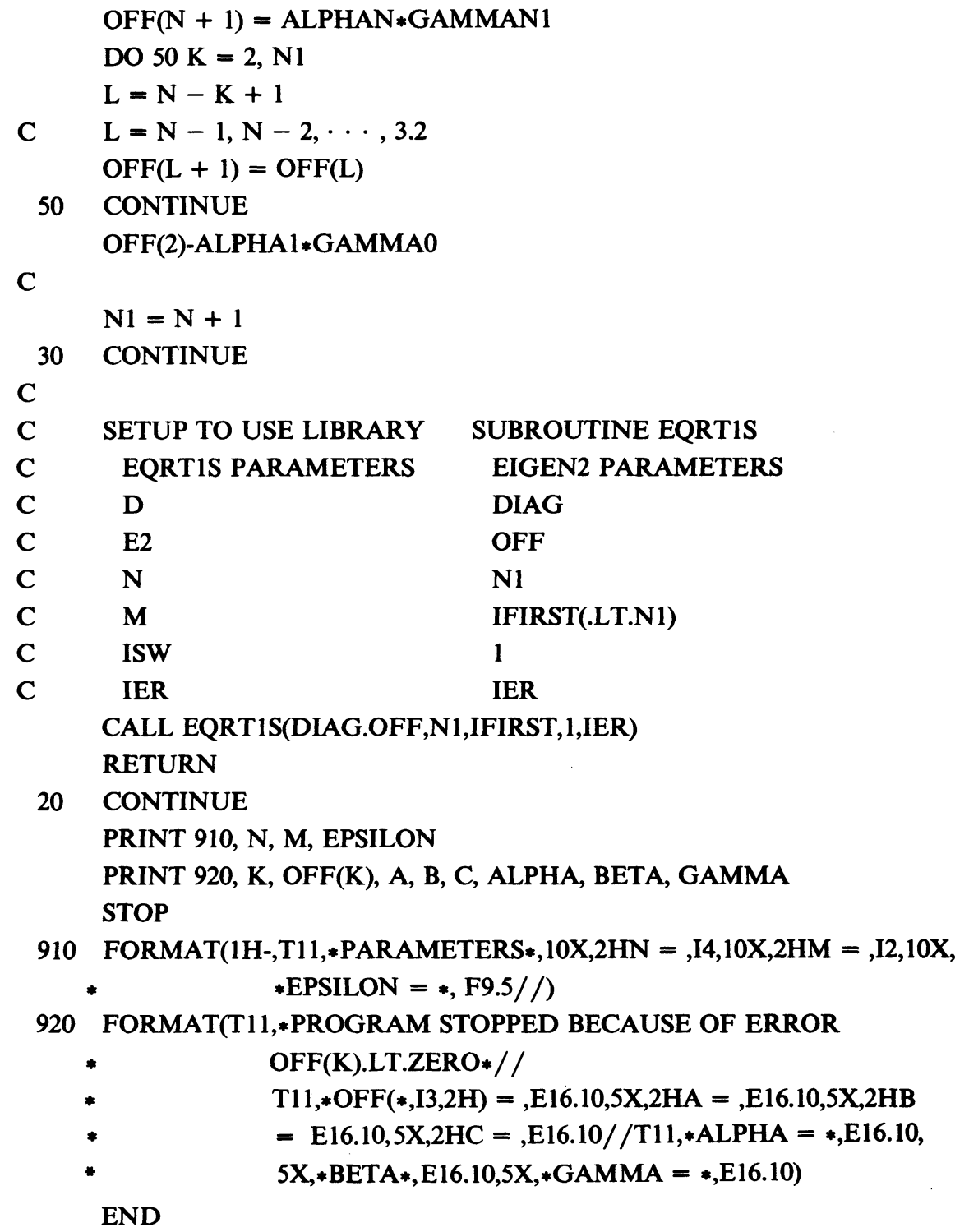

\section{References}

[1] A. L. Besse, Manifolds all of whose geodesics are closed, Ergebnisse Math. und Grenzgebiete, Vol. 93, Springer, Berlin, 1978.

[2] E. Isaacson \& H. B. Keller, Analysis of numerical method, Wiley, New York, 1966.

[3] N. J. Vilenkin, Special functions and the theory of group representations, Transl. Math. Monographs, Vol. 22, Amer. Math. Soc., 1968. 
[4] F. W. Warner, Foundations of differentiable manifolds and Lie groups, Scott, Forman \& Co., Glenview, IL, 1971.

[5] Alan Weinstein, Application des opérateurs intégraux de Fourier aux spectres des variétés riemanniennes, C. R. Acad. Sci. Paris, Sér. A, 279 (1974) 229-230.

[6] __ Asymptotics of eigenvalue clusters for the Laplacian plus a potential, Duke Math. J. 44 (1977) 883-892.

[7] O. Zoll, Ueber Flächen mit Scharen geschlossener geodätischen Linien, Math. Ann. 57 (1903) $108-133$.

HOWARD UNIVERSITY 
